\title{
Prospecção Científica e Tecnológica da Tuberculose no Maranhão e o Uso Medicinal da Copaifera Langsdorffii no Tratamento
}

\author{
Scientific and Technological Prospecting for Tuberculosis in Maranhão \\ and the Medicinal Use of Copaifera Langsdorffii in Treatment
}

\author{
Vitória Santos Azevedo ${ }^{1}$ \\ Adrielle de Sá Araújo ${ }^{1}$ \\ Marcelo Borges de Sousa ${ }^{1}$ \\ Marcos dos Santos Barros ${ }^{1}$ \\ Jefferson Almeida Rocha ${ }^{2}$ \\ ${ }^{1}$ Universidade Federal do Maranhão, Campus Grajaú, MA, Brasil \\ ${ }^{2}$ Universidade Federal do Maranhão, Campus de São Bernardo, MA, Brasil
}

\begin{abstract}
Resumo
A Tuberculose é a doença que mais mata no mundo, possuindo um alto índice de prevalência e de mortalidade. Sendo assim, este trabalho tem como finalidade a realização da prospecção científica e tecnológica de pesquisas sobre a tuberculose no Maranhão e o uso medicinal da planta Copaifera langsdorffii contra a doença. Na busca de patentes, foram utilizadas as bases: INPI, Espacenet, USPTO e Web of Science; e os artigos nas bases: Scielo, Bireme, PubMed, Web of Science, Scopus, Science Direct, ACS e Google acadêmico. Os resultados na busca de patentes sobre a tuberculose no Maranhão e o uso medicinal da planta copaíba foram negativos em relação a patentes publicadas, e, em relação aos artigos, o resultado foi bastante diferente, obteve-se uma grande quantidade de pesquisas relacionadas à tuberculose no Maranhão e ao uso da copaíba para o tratamento da tuberculose. É visível o incentivo para o aumento de pesquisas nessa área comprovando a veracidade da planta para o tratamento da tuberculose.
\end{abstract}

Palavras-chave: Tuberculose. Maranhão. Copaíba.

\begin{abstract}
Tuberculosis is the disease that kills the most in the world, with a high prevalence and mortality rate. This work aims to carry out scientific and technological research on tuberculosis in Maranhão and the medicinal use of the Copaifera langsdorffii plant against the disease. In the search for patents, we used the databases: INPI, Espacenet, USPTO and Web of Science, and articles in the databases: Scielo, Bireme, PubMed, Web of Science, Scopus, Science Direct, ACS and Google Scholar. The results in the search for patents on tuberculosis in Maranhão and the medicinal use of the copaiba plant were a negative result of published patents, in relation to articles the result was quite different, with a large number of articles related to tuberculosis in Maranhão and the use of copaiba in the treatment of tuberculosis. It is visible the incentive to increase research in this area proving the veracity of the plant against tuberculosis.
\end{abstract}

Keywords: Tuberculosis. Maranhão. Copaiba.

Área Tecnológica: Prospecção Científica. Prospecção Tecnológica. 


\section{Introdução}

Um terço da população do mundo, num total de dois bilhões de pessoas, está infectada com Mycobacterium tuberculosis. Entre essa população, cerca de 9,27 milhões de pessoas ficam doentes e dois milhões morrem a cada ano, incluindo 460.000 contaminados com o vírus da imunodeficiência humana (HIV). O Brasil tem o $16^{\circ}$ maior número de casos de tuberculose e possui a maior taxa de incidência (COSTA et al., 2019).

Antes da chegada dos exploradores europeus, a tuberculose tinha sido relatada em populações ativas americanas e em populações brasileiras, sendo detectada a partir do período colonial do Rio de Janeiro, mostrando infecção por tuberculose em indivíduos de ascendência predominantemente europeia, bem como de escravos africanos. Não tendo procedências de estudos demonstrando infecção por tuberculose em brasileiros natos, a história e o cenário epidemiológico da tuberculose no Brasil ainda são desconhecidos (GUEDES et al., 2018).

A Tuberculose é uma doença infecciosa causada pela Mycobacterium tuberculosis ou Bacilo de Koch, essa bactéria é aeróbica estrita, ácido-resistente de desenvolvimento lento. É o agente infeccioso que mais mata no mundo, possuindo um alto índice de prevalência e mortalidade. Essa doença afeta com mais frequência os pulmões, mas pode infectar qualquer parte do corpo, dos gânglios ao sistema nervoso central (GROSCH et al., 2015). A transmissão do agente é feita por meio do ar, pela emissão de gotículas, de pessoa a pessoa, sendo que a tosse se caracteriza como o principal meio propagador. O quadro clínico dos portadores de tuberculose apresenta um comprometimento do estado geral, como febre baixa, geralmente vespertina, além de episódios de sudorese noturna, inapetência e emagrecimento, dor torácica e tosse, inicialmente seca, e, quando produtiva, acompanhada ou não de escarros hemoptoicos (MORAES et al., 2017).

O diagnóstico da tuberculose no Brasil é realizado por meio da avaliação clínica radiológica dos pulmões e de outros testes, como o teste tuberculínico (PPD), a detecção dos bacilos álcool-ácido resistentes (BAAR) em amostras de escarro pela coloração direta (Ziehl-Neelsen) ou por cultura microbiológica das amostras (ZAGMIGNAN et al., 2014).

Para prevenir a tuberculose, utiliza-se a vacina BCG (elaborada a partir do bacilo de Calmette e Guérin - BCG) e, no tratamento da infecção latente por tuberculose (ILTB), é utilizado o medicamento isoniazida (TPI). O fornecimento de medicamentos antituberculose é gratuito, não está disponível comercialmente, e esse medicamento é distribuído apenas por redes de saúde pública, pois são entregues aos pacientes com apresentação da ficha de notificação devidamente preenchida (RABAHI et al., 2017). A tuberculose é uma doença cujas causas, formas de contágio, prevenção e, sobretudo, práticas de cuidado são envolvidas em diferentes concepções por boa parte da sociedade, pouco conhecidas e construídas ao longo dos anos (SILVA; SOUSA; SANT’ANNA, 2014).

Mesmo na atualidade, a tuberculose permanece ocupando destaque entre as principais doenças infectocontagiosas em países como o Brasil. Desafios como a epidemia da infecção pelo vírus da imunodeficiência humana (HIV), a tuberculose multirresistente (MR-TB) e extensivamente resistente (XDR-TB), a transição epidemiológica com o aumento de doenças não transmissíveis (DNT) e a urbanização acelerada são variáveis que favorecem a persistência da tuberculose na forma epidêmica fazendo com que essa doença cresça a cada dia (OMS, 2015). 
Entre os 22 países que concentram $80 \%$ dos casos de tuberculose registrados mundialmente, o Brasil ocupa o $19^{\circ}$ lugar em incidência de casos; e, no ranque mundial, o país ocupa a $108^{a}$ posição em incidência de casos. Em 2010 foram notificados aproximadamente 71 mil casos de tuberculose, entre esses casos, 4,8 mil pessoas faleceram, ou seja, esses números constituem-se na terceira causa de óbitos por doenças infecciosas (NETO et al., 2012).

Em dados obtidos em 2015 pelo Sistema de Informação de Agravos de Notificação (SINAN) e pela Secretaria Estadual de Saúde (SES), no Estado do Maranhão foram notificados 1.763 casos no ano de 2014, estabelecendo-se, assim, nos últimos cinco anos, em $4^{\circ}$ lugar entre os estados do Nordeste que apresentam as maiores taxas de incidências de tuberculose por 100 mil habitantes. Tal configuração faz com que a tuberculose seja uma das principais causas de morbimortalidade, atingindo indistintamente faixas etárias e classes sociais (GROSCH et al., 2015).

No Estado do Maranhão, oito municípios são citados como prioritários: São Luís, Açailândia, Caxias, Codó, Imperatriz, Paço do Lumiar, São José de Ribamar e Timon, com uma cobertura de $29 \%$ das unidades de saúde com o Programa Nacional de Controle da Tuberculose (PNCT) implantado. Dessas unidades, $45 \%$ utilizam a estratégia de tratamento supervisionado (MORAES et al., 2017).

As mudanças mais recentes e importantes na epidemiologia da tuberculose estão associadas à epidemia do vírus da imunodeficiência humana (HIV) como também ao surgimento da resistência ao uso dos fármacos de tuberculostáticos, por este motivo é essencial que haja, imediatamente, pesquisas que comprovem a eficácia de outros fármacos. (SILVA; SOUSA; SANT'ANNA, 2014). A infecção pelo HIV agrava a epidemia da tuberculose, seja por aumentar a vulnerabilidade à infecção pela Mycobacterium tuberculosis, seja por torná-la uma doença altamente ativa (CARVALHO et al., 2006).

A resistência ao uso dos tuberculostáticos e o surgimento de novos agravantes têm levado pesquisadores ao conhecimento e ao aprofundamento sobre o uso de plantas na medicina tradicional, sendo elas importantes fontes para a obtenção de novas substâncias de interesse biológico. A descoberta destes compostos tem sido impulsionada por pesquisa etnobiológica sobre o uso da biodiversidade (RIBEIRO et al., 2014).

Os medicamentos que são fabricados a partir de fontes naturais, com microrganismos e extratos de plantas, são um fator de investigação frequente para a descoberta de compostos antimicrobianos, como a copaíba (Copaifera langsdorffii), cujo óleo está sendo objeto de pesquisas para obter um grau de eficiência no tratamento contra a tuberculose. Essa planta também é muito utilizada na medicina popular brasileira, devido às suas aplicações farmacológicas comprovadas em uso popular. Esse óleo pode apresentar composição diversa, dependendo da sua fonte botânica (SILVA et al., 2017).

A utilização do óleo de copaíba foi difundida no século XVII, quando os primeiros médicos residentes no Brasil tentavam contornar a escassez de remédios cujo suprimento à Colônia era irregular. A copaíba, ou copaibeira, é uma árvore de grande porte da família Leguminosae encontrada em todo o Brasil. O gênero Copaifera possui mais de 25 espécies, sendo a maioria encontrada na América do Sul. Comumente são chamadas de copaíba, pau-d'óleo, copaíba-roxa e copaíba-mari-mari (MENDONÇA; ONOFRE, 2009).

O óleo de copaíba é obtido a partir de uma incisão no tronco, o qual produz um óleo viscoso rico em sesquiterpenos (fração volátil) e diterpenos (fracção resinosa), conhecido como 
copaíba branco (SOARES et al., 2003), sendo constituído de dois grupos de substâncias distintas, solúveis entre si, um deles constituído de substâncias voláteis, correspondendo a cerca de $90 \%$ de massa do óleo resina e o outro grupo constituído de substâncias não voláteis (DEUSz et al., 2011), sendo essas substâncias o ácido copálico, e os sesquiterpenos $\beta$-cariofileno e o $\alpha$-copaeno, que são os componentes fundamentais do óleo, encontrando-se em todos os óleos de copaíba até hoje estudados. Talvez possam vir a ser usados como biomarcadores para a autenticidade desses óleos (SOARES et al., 2003).

Os óleos de copaíba são importantes medicamentos utilizados principalmente pela sua cura $e$ atividades anti-inflamatórias. Este óleo tem composição variável, a qual, de acordo com a literatura, pode ser originária de vários fatores; e pode ser futuramente utilizado no tratamento da tuberculose (BARBOSA et al., 2012). Com isso, este trabalho tem por objetivo o desenvolvimento de uma prospecção científica e tecnológica da tuberculose no Maranhão e, dessa forma, pretende apresentar os estudos do uso da copaíba no tratamento da tuberculose.

\section{Metodologia}

Para a realização da pesquisa, foram feitas buscas em bancos de dados de patentes para o estudo prospectivo tecnológico e científico em bases de dados nacionais e internacionais para a realização da prospecção.

Para a realização da prospecção tecnológica, foram realizadas buscas nos bancos de dados de patentes: Instituto Nacional da Propriedade Industrial (INPI), Europe's Network of Patents databases (Espacenet), United States Patent and Trademark Office (USPTO) e Thompson Reuters (Web of Science), utilizando-se como objeto de pesquisa as palavras: "tuberculosis and maranhão", "tuberculosis and copaíba and maranhão", "tuberculosis and copaíba", "maranhão and copaíba".

A prospecção científica foi feita nas seguintes bases de dados: Scientífic Eletronic Library Online (Scielo), Portal Regional da Biblioteca Virtual em Saúde (Bireme), Nacional Center for Biotechnology information (PubMed), Thompson Reuters (Web of Science), grupo Elsevier Group (Scopus), Science Direct, American Chemical Society (ACS) e Google acadêmico. Os descritores utilizados para a pesquisa foram: "mycobacteruim tuberculosis and Maranhão", "copaifera langsdorffii and mycobacteruim tuberculosis", "copaifera langsdorffii and tuberculosis", "tuberculosis and Maranhão", "tuberculosis and copaíba". Utilizaram-se todos os termos e/ou títulos relacionados que serviriam de base para a realização e montagem da pesquisa, lembrando-se que em alguns sites escreveu-se "tuberculosis" e em outros "tuberculose", para bases em inglês e português, respectivamente.

Os dados prospectados foram obtidos entre abril e maio de 2019 e também foram analisados os pedidos de patentes e publicações de artigos sem uma restrição de data para início até o ano de 2018. Além das palavras-chave, a pesquisa foi realizada com a utilização de alguns recursos, como as aspas, para ajudar a obter o maior número possível de documentos. Depois dos resultados obtidos nas buscas de dados pesquisados, foi realizada uma comparação entre os artigos a fim de se avaliar o andamento das pesquisas sobre a tuberculose no Brasil, com ênfase no Estado do Maranhão, e o uso medicinal da copaíba no tratamento desta infecção. 
A seguir apresenta-se o organograma (Figura 1) das bases de dados usadas para as pesquisas e seus respectivos descritores.

Figura 1 - Organograma das bases utilizadas nas prospecções científicas e tecnológicas

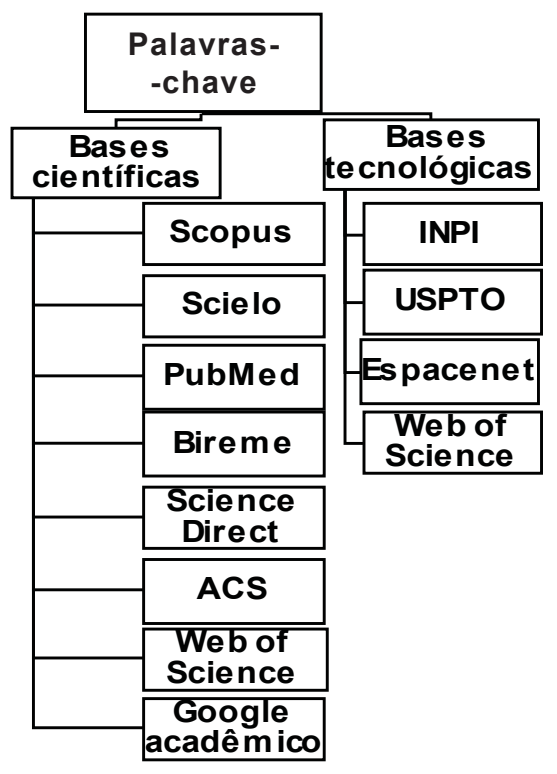

Fonte: Elaborada pelos autores deste artigo (2019)

\section{Resultados e Discussão}

Nesta seção serão apresentadas tabelas e figuras de modo a representar não somente os resultados como também as discussões acerca do que foi obtido. De imediato, percebe-se que é visível a discrepância dos resultados obtidos entre a prospecção tecnológica e a prospecção cientifica, já que há mais produções de artigos do que pedidos de patentes. Isso se deve a vários fatores que serão apresentados a seguir.

\subsection{Prospecção Tecnológica}

Perante o resultado negativo nas buscas de patentes sobre tuberculose e o uso medicinal da copaíba como forma de tratamento, observou-se a grande necessidade de estudos e de pesquisas sobre a planta copaíba no tratamento da tuberculose, para que assim haja a fabricação de mais patentes e também o aprofundamento no conhecimento medicinal da planta contra a Mycobacterium Tuberculosis, pois, de acordo com a literatura, o uso de extratos vegetais e fitoquímicos com fins medicinais é uma das mais antigas formas de prática medicinal utilizada pela humanidade (GONÇALVES; FILHO; MENEZES, 2005). 
Tabela 1 - Depósitos de patentes sobre a tuberculose no Maranhão pesquisados nas bases de dados da INPI, Espacenet, USPTO e Web of Science e seus respectivos descritores

\begin{tabular}{ccccc} 
PalaVRAS-CHAVE & INPI & USPTO & ESPACENET & WeB OF SCIENCE \\
Tuberculosis and Maranhão & 0 & 0 & 0 & 0 \\
\hline Tuberculosis and Copaiba and Maranhão & 0 & 0 & 0 & 0 \\
\hline Tuberculosis and Copaiba & 0 & 0 & 0 & 0 \\
\hline Maranhão and Copaiba & 0 & 0 & 0 & 0 \\
\hline
\end{tabular}

Fonte: Elaborada pelos autores deste artigo (2019)

A baixa taxa de publicação de depósitos de patentes sobre a tuberculose pode ser reflexo das políticas de incentivo que se encontram sem tempo para amadurecerem, é possível citar o caso da Lei de inovação e o baixo investimento de empresas públicas e privadas, além do extenso tempo de tramitação do registro de patentes, fazendo com que pesquisadores e empresas depositem patentes em países em que o aceite seja mais rápido, resultando assim em um número insatisfatório de patentes publicadas, relacionadas à tuberculose, no Brasil (SOUZA, 2015). A tuberculose faz parte do rol das doenças negligenciadas, um grupo de afecções transmissíveis, cujo tratamento é inexistente, precário ou desatualizado. Diante desses fatores, há necessidade de investimentos tecnológicos em conhecimentos e em pesquisas para acelerar o progresso da eliminação da tuberculose (PEDRO et al., 2014).

Muitos fatores podem ter agravado a incidência de tuberculose no Maranhão, entre eles: o aumento de pessoas diabéticas, a baixa imunidade, a situação socioeconômica da população, resultando em dificuldades de acesso aos serviços de saúde, o crescimento da população de marginais (a população privada de liberdade facilita a ocorrência e transmissão da tuberculose). Várias situações têm sido atribuídas a esse quadro, como à não adesão, que abrange barreiras sociais e culturais, e problemas inerentes aos medicamentos (SILVA; SOUSA; SANT'ANNA, 2014).

Somente no ano de 2014 no Maranhão, foram notificados 163 óbitos por tuberculose, perfazendo um grau de mortalidade de 2,4/100.000 habitantes, sendo que, no total desses óbitos, a forma da tuberculose pulmonar representou $86,5 \%$ dos casos. No Maranhão, foram verificados ainda 57 óbitos nos quais a tuberculose aparece como principal causa associada e, desse total, $32(56,1 \%)$ apresentaram a Aids como a causa básica desses óbitos. Vale salientar que 124 óbitos com tuberculose como causa básica ou associada não estavam notificados no SINAN (BRASIL, 2016).

Nesse contexto, é mais que necessário o aprofundamento em estudos sobre a tuberculose no Maranhão, pois, de acordo com os dados, é possível analisar que o número de casos é elevado, fazendo com que pessoas que não tenham o conhecimento da doença demorem para se tratar, pois não possuem noção sobre ela e nem sobre os métodos de seu tratamento. Por esse mesmo motivo o presente estudo se faz necessário, pois é visível que haja inovação em relação a esse tema tão presente e ao mesmo tempo tão pouco debatido. Mesmo com tantos programas de prevenção e de tratamento da tuberculose, essa ainda é uma doença endêmica no Estado.

Em dados obtidos pelo Sistema de Informação de Agravos de Notificação encontram-se os números de casos de tuberculose no Estado do Maranhão no período de 2008 a 2017 (Figura 
2). No ano de 2008, houve uma grande taxa de incidência de casos de tuberculose notificados, sendo perceptível a permanência desses altos índices no decorrer dos anos, no entanto, vale ressaltar que no ano de 2014 houve uma diminuição de 533 casos notificados e, a seguir, teve um aumento no decorrer dos anos seguintes, sendo o ano de 2017 com o registro de 2.095 casos notificados de pacientes com tuberculose.

Figura 2 - Índice de casos registrados de tuberculose no Maranhão nos últimos 10 anos

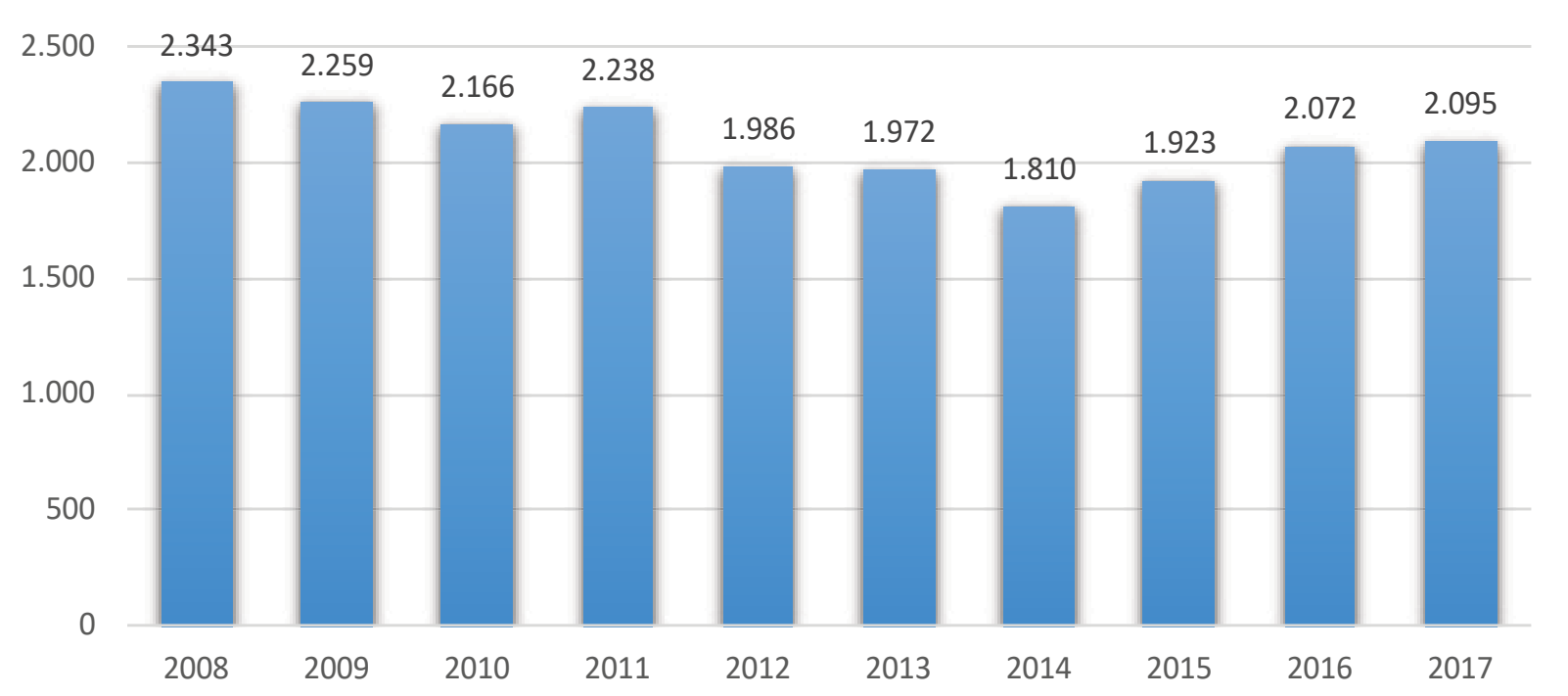

Fonte: Elaborada pelos autores deste artigo com base em SINAN (2018)

A falta de patentes farmacêuticas relacionadas à tuberculose e o uso medicinal da copaíba ou outros medicamentos como forma de tratamento se dão por conta da preocupação quanto aos efeitos nocivos relacionados à imposição ao acesso a tratamentos mais eficazes como também as consequências que podem surgir para a saúde pública (MEINERS, 2008). Além disso, a falta de patentes transcende uma falha nos avanços tecnológicos, fazendo com que não haja um avanço em relação a novas formas de tratar a tuberculose e ao uso do óleo de copaíba como forma de tratamento. A tuberculose possui uma grande resistência aos fármacos, acrescida da grande variação de efeitos colaterais dos medicamentos utilizados para o seu tratamento, tornando necessária a pesquisa e a produção de novos fármacos que ajam de maneira eficaz contra a doença. Diante disso, algumas pesquisas apontam a ação do óleo da copaíba contra a tuberculose (FREIRE; WOLTER; PEREIRA JÚNIOR, 1997).

\subsection{Prospecção Científica}

Os números da prospecção científica foram discrepantes em relação à quantidade de patentes encontradas, obtendo-se o maior número de artigos na base de dados do Google acadêmico, com um total de 20.826 artigos encontrados, seguido do Science Direct com 175 e Web of Science com 152 artigos, tendo as outras bases de dados apresentado resultados bem menores de artigos científicos, como é possível averiguar na Tabela 2. 
Tabela 2 - Quantidade de artigos encontrados e seus respectivos descritores

\begin{tabular}{ccccccccc}
\hline Palavras-chave & Scopus & Bireme & Pubmed & Scielo & $\begin{array}{c}\text { Science } \\
\text { Direct }\end{array}$ & $\begin{array}{c}\text { Web of } \\
\text { Science }\end{array}$ & Acs & $\begin{array}{c}\text { Google } \\
\text { Acadêmico }\end{array}$ \\
$\begin{array}{c}\text { Mycobacterium } \\
\text { tuberculosis and } \\
\text { maranhão }\end{array}$ & 4 & 16 & 8 & 2 & 28 & 8 & 0 & 14.000 \\
$\begin{array}{c}\text { Copaifera langsdorffii } \\
\text { and Mycobacterium } \\
\text { tuberculosis }\end{array}$ & 1 & 0 & 0 & 1 & 1 & 1 & 0 & 186 \\
$\begin{array}{c}\text { Copaifera langsdorffii } \\
\text { and tuberculosis }\end{array}$ & 2 & 0 & 0 & 1 & 9 & 1 & 0 & 1.270 \\
$\begin{array}{c}\text { Tuberculosis and } \\
\text { maranhão }\end{array}$ & 15 & 26 & 24 & 14 & 107 & 10 & 3 & 4.110 \\
\hline $\begin{array}{c}\text { Tuberculosis } \\
\text { and copaîba }\end{array}$ & 1 & 2 & 0 & 1 & 30 & 132 & 0 & 1.260 \\
\hline
\end{tabular}

Fonte: Elaborada pelos autores deste artigo

Em relação aos resultados, nota-se quão alta é a quantidade de artigos encontrados, porém ao analisar uma certa quantidade deles, foi possível perceber que vários se repetem nas diversas bases de dados pesquisadas. Na base do Google acadêmico, por exemplo, a maioria dos artigos encontrados é facilmente encontrada nas outras bases. É de suma importância salientar também que, assim como alguns artigos se repetem, outros possuíam apenas algum termo/palavra relacionados à pesquisa, como por exemplo, artigos apresentavam o termo "copaíba" e/ ou "tuberculose", mas não tratavam da temática proposta. Por isso tais resultados tornaram-se inconsistentes (Figura 3).

Figura 3 - Depósito de artigos referentes à tuberculose, à Copaifera langsdorffii e à Tuberculosis and copaíba

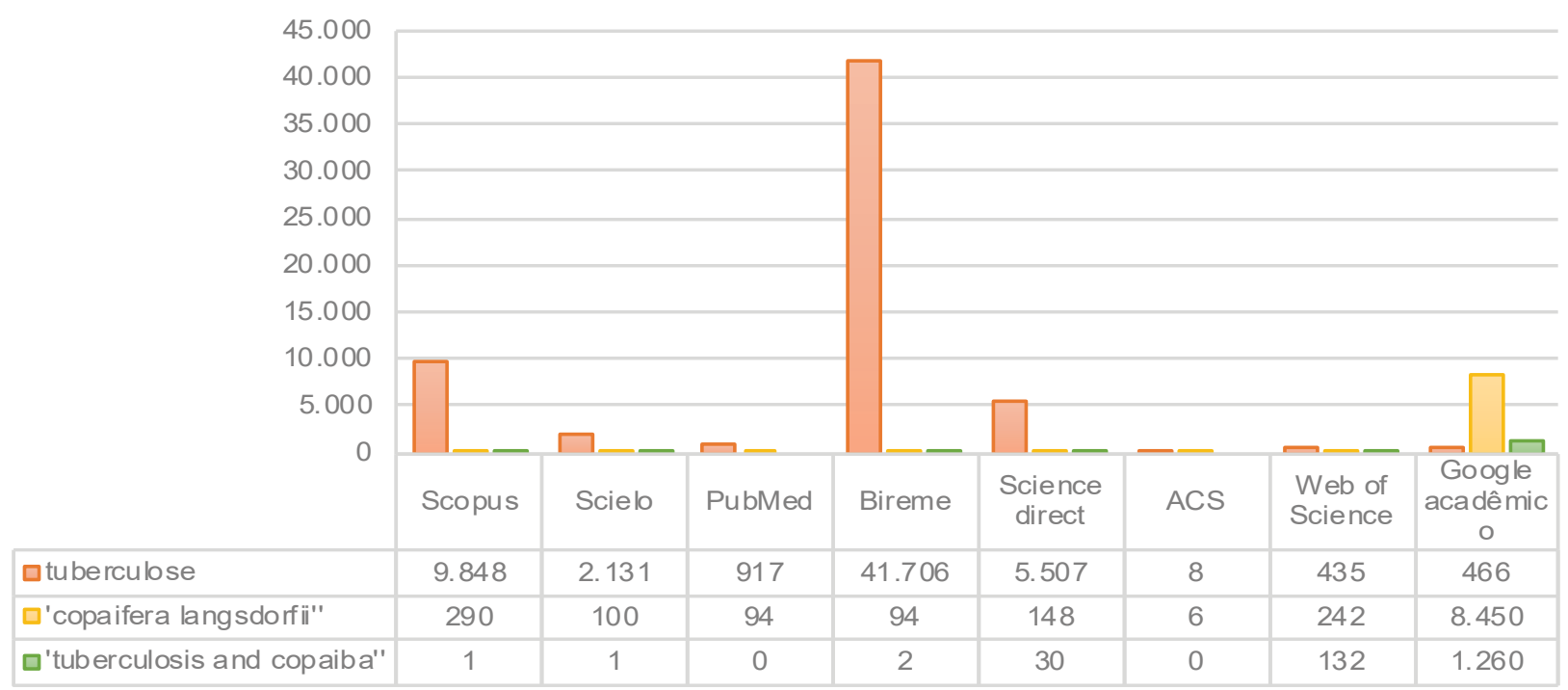

Fonte: Elaborada pelos autores deste artigo

As informações da Figura 3 mostram uma comparação dos artigos encontrados nessas bases de dados com seus respectivos resultados, sendo que há um grande número de artigos relacionado à tuberculose, em um total de 61.018; e sobre a copaíba tem-se o equivalente a 
9.424 artigos, porém o resultado muda quando se usa o descritor "tuberculosis and copaíba", que obtém somente 1.426 artigos, ou seja, um resultado irrelevante aos números superiores de artigos adquiridos. Sendo assim, isso mostra a escassez de publicações sobre a tuberculose e o uso medicinal da copaíba no tratamento, e mesmo possuindo um alto número de artigos encontrados utilizando-se apenas um dos termos (tuberculose ou copaíba), quando se faz a junção dos dois termos os resultados são inconcebíveis.

Os artigos científicos são um meio de divulgação de conhecimento científico (MUELLER; PERUCCHI, 2014) e, mesmo com um número considerável de artigos encontrados nessas bases de dados, é preciso o incentivo para a produção de novos trabalhos, pois a busca de literatura científica como meio de estudo e de desenvolvimento leva o leitor a produzir novas pesquisas relacionadas à tuberculose e novas formas de tratamento dessa doença (KIRCHHOF; LACERDA, 2012), entre elas a produção de medicamentos naturais que possam ser utilizados no seu tratamento, como é o caso do óleo extraído da árvore do gênero Copaífera, a copaíba, que tem sido utilizada por mais de 500 anos na medicina tradicional popular, e por possuir uma grande diversidade de aplicações, como: antitumoral, anti-inflamatória, antioxidante, antimicrobiana (TINCUSI et al., 2002), contra uma grande diversidade de microrganismos e cicatrizante sobre diferentes tecidos do corpo. Estudos têm sido realizados para comprovar as atividades medicinais conferidas ao óleo e para a indicação científica desse óleo à terapêutica de várias doenças (PIERI; MUSSI; MOREIRA, 2009).

Porém, levando-se em consideração as diversas indicações do óleo (MACIEL; PINTO; VEIGA JUNIOR, 2002), os estudos sobre a copaíba sendo empregada no tratamento da tuberculose têm-se apresentado ainda restritos e escassos. No entanto, surge ainda a possibilidade de estudar as atividades das diferentes frações componentes do óleo da copaíba, o que se reverteria na formação de alternativas terapêuticas para muitas patologias humanas, principalmente no seu uso para tratar a tuberculose (PIERI; MUSSI; MOREIRA, 2009).

Segundo Silva et al. (2018), em suas pesquisas para o tratamento da tuberculose a partir de compostos naturais isolados do óleo da copaíba, os resultados obtidos foram promissores contra a linhagem bacteriana da tuberculose. Em seus resultados, os autores citam que os diterpenos naturais podem ser considerados como promissores para novas investigações da atividade antituberculosa, tanto como potencial de fonte natural ou como protótipos de novos compostos ativos. Os resultados obtidos dão origem a algumas considerações futuras, pesquisas que buscarão compostos ativos contra Mycobacterium tuberculosis, especialmente entre os diterpenos encontrados no óleo da copaíba (SILVA et al., 2017).

Nos últimos anos, o retorno à terapêutica natural trouxe de volta os fitoterápicos para as farmácias de todo o país, mas o conhecimento de seu uso e suas aplicações é pouco, ou é bastante confuso nas centenas de publicações de artigos que não apresentam mais que duas ou três propriedades farmacológicas bastante conhecidas do óleo da copaíba (VEIGA JUNIOR; PINTO, 2012). Mas após anos sem o surgimento de novas drogas contra a tuberculose, uma substância extraída do óleo da copaîba (Copaifera langsdorffii), planta originária da Amazônia, poderá vir a ser a base de um fitomedicamento a ser usado no tratamento da doença (FREIRE; WOLTER; PEREIRA JÚNIOR, 1997). Pois tem-se conhecimento de que a tuberculose é uma doença em livre expansão no mundo e faltam novas drogas que substituam o atual arsenal de antibióticos, rifampicina, isoniazida e etambutol, no tratamento das cepas resistentes de Mycobacterium tuberculosis (GILBERT, 2006). 
Diante das propriedades do óleo da planta, a copaíba, e o seu efeito no tratamento da tuberculose, é plausível a necessidade de investimentos em conhecimentos e em pesquisas para acelerar o progresso da eliminação da tuberculose. Nesse sentido, os artigos utilizados nas prospecções científicas publicados sobre aspectos relevantes da doença têm por objetivo apontar algumas de suas nuances visando à atualização literária e à busca de um olhar mais atento à problemática da tuberculose no Maranhão (PEDRO et al., 2014).

A área de foco de informação sobre a tuberculose e a planta copaíba compreende o conjunto de ações com a finalidade de garantir o acesso, o uso, a produção e a disseminação da informação com qualidade, de modo a avançarem em áreas de atuação e campos de conhecimento científico, com relevantes impactos sociais (REIS et al., 2017), sabendo-se que a inovação é um fator fundamental para garantir o desenvolvimento de regiões e países (MARTINS; GALINA, 2010).

\section{Considerações Finais}

O Brasil tem o $16^{\circ}$ maior número de casos de tuberculose e possui a maior taxa de incidência dessa doença (COSTA et al., 2019). Com relação ao Estado do Maranhão, entre os anos de 2008 a 2017, o SINAN registrou 20.864 casos de tuberculose notificados, tendo um alto número desses casos no ano de 2008, em um total de 2.343 casos. Assim, são necessárias pesquisas em relação ao estudo dessa doença com enfoque em um determinado medicamento natural que possa ser utilizado no seu tratamento, visando também um maior número de publicações de artigos e patentes, o que torna necessária a realização de políticas de incentivo à pesquisa, pois quanto mais pesquisas, mais avanços se alcançarão em relação a essa doença, que atinge várias pessoas no Maranhão, no Brasil e no mundo.

Com base nos resultados obtidos na busca de patentes sobre a tuberculose no Maranhão e o uso medicinal da planta copaíba, apresentou-se um resultado negativo de patentes publicadas, mas em relação aos artigos o resultado foi bastante diferente, tendo-se encontrado uma grande quantidade deles relacionados à tuberculose no Maranhão e ao uso da copaíba no seu tratamento.

Em relação ao estudo do presente artigo, foi possível compreender que ainda são poucas as publicações de artigos e patentes, tendo em vista que a tuberculose é uma doença de alto índice, e isso mostra a necessidade de que haja produção de novos artigos e de patentes relacionadas à tuberculose no Maranhão, pesquisas sobre os diversos tipos de tuberculose, sua forma de contágio, aspectos clínicos e, principalmente, as formas de tratamento, tornando-se eficaz a realização de pesquisas de cunho cientifico e tecnológico sobre a planta de uso medicinal, vulgarmente chamada de copaíba.

Conclui-se que as várias publicações mundiais, na maioria, mostram preocupação sobre tudo que envolve essa doença, entretanto no Maranhão as publicações são escassas, o que do mesmo modo acontece com as pesquisas relacionadas a novos meios de combate a essa doença. Uma vez que, de acordo com as informações deparadas, a tuberculose possui uma grande resistência aos fármacos, por este mesmo motivo tornam-se necessárias a pesquisa e a produção de novos fármacos que atuem de maneira eficaz contra a tuberculose. Diante disso, pesquisas apontam a ação do óleo da copaíba contra a tuberculose, pois este possui diterpenos 
naturais que podem ser considerados como promissores para novas investigações da atividade antituberculosa, tanto como potencial fonte natural ou como protótipo de novos compostos ativos (SILVA et al., 2017), sendo que pode vir a ser utilizado com uma nova forma de tratamento contra a tuberculose, se comprovada sua eficácia (FREIRE; WOLTER; PEREIRA JÚNIOR, 1997).

\section{Referências}

ARAUJO, K. S. et al. Prospecção científica e tecnológica da dengue no Brasil e no Maranhão no período de 1994 a 2014. Cadernos de Prospecção, Salvador, v. 9, n. 3, p. 313-322, 2016.

BARBOSA, P. C. S. et al. Influence of Abiotic Factors on the Chemical Composition of Copaiba Oil (Copaifera multijuga Hayne): Soil Composition, Seasonality and Diameter at Breast Height. J. Braz. Chem. Soc., [S.I.], v. 23, n. 10, p. 1.823-1.833, 2012.

BRASIL. Ministério da Saúde. Secretaria de Vigilância em Saúde. Departamento de Vigilância das Doenças Transmissíveis. Panorama da tuberculose no Brasil: a mortalidade em números. Brasília: Ministério da Saúde, 2016. 46p.

BRASIL. Ministério da Saúde. Secretaria de Vigilância em Saúde. Sistema de Informação de Agravos de Notificação. Série histórica do número de casos novos de tuberculose - Brasil, Regiões e Unidades Federadas de residência por ano diagnóstico (1990 a 2017*). Brasília, 2018.

CARVALHO, L. G. et al. Co-infection with Mycobacterium tuberculosis and human immunodeficiency virus: an epidemiological analysis in the city of Taubaté, Brazil. Jornal Brasileiro Pneumol., [S.l.], v. 32, n. 5, p. 424-429, 2006.

COSTA, M. R. et al. Characteristics of basic health units and detection of tuberculosis cases. Journal of the Brazilian Society of Tropical Medicine, Uberaba, [S.l.], v. 52, n. 20, p. 1-9, 2019.

DEUS, R. J. A.; ALVES, C. N.; ARRUDA, M. S. P. Avaliação do efeito antifúngico do óleo resina e do óleo essencial de copaíba (Copaifera multijuga Hayne). Rev. Bras. Plantas Med., Botucatu, SP, v. 13, n. 1, 2011.

FREIRE, S. M. L.; WOLTER, E. L. A.; PEREIRA JÚNIOR, O. L. Avaliação da ação tuberculocida de plantas da Amazônia. Repositório do INPA, Manaus, p. 147-148, 1997.

GILBERT, B. Industrializable Natural Products from the Amazon. Revista Fitos, [S.l.], v. 2, n. 3, dez. 2006.

GONÇALVES, A. L.; FILHO, A.; MENEZES, H. Comparativo da atividade antimicrobiana de extratos de algumas árvores nativas. Arq. Inst. Biol., São Paulo, v. 72, n. 3, p. 353-358, jul.-set. 2005.

GROSCH, C. A. et al. Prevalência da tuberculose no Maranhão. Rev. Investig, Bioméd., São Luís, v. 7, p. 28-34, 2015.

GUEDES, G. et al. Tuberculosis in post-contact Native Americans of Brazil: Paleopathological and paleogenetic evidence from the Tenetehara-Guajajara. PLoS ONE, [S.l.], v. 13, n. 9, 2018.

KIRCHHOF, A. L. C.; LACERDA, M. R. Desafios e perspectivas para a publicação de artigos - Uma reflexão a partir de autores e editores. Texto Contexto Enferm., Florianópolis, v. 21, n. 1, p. 18593, jan.-mar., 2012. 
MACIEL, M. A. M.; PINTO, A. C.; VEIGA JUNIOR, V. F. Plantas medicinais: a necessidade de estudos multidisciplinares. Química Nova, [S.I.], v. 25, n. 3, p. 429-438, 2002.

MARTINS, D. S.; GALINA, S. V. R. Modelos de avaliação de instrumentos públicos de incentivos à inovação tecnológica. [S.l.]: Enegep, 2010.

MENDONÇA, D. E.; ONOFRE, S. B. Atividade antimicrobiana do óleo-resina produzido pela copaiba - Copaifera multijuga Hayne (Leguminosae). Revista Brasileira de Farmacognosia Brazilian Journal of Pharmacognosy, [S.l.], v. 19, n. 2b, p. 577-581, abr.-jun., 2009.

MEINERS, C. M. M. A. Patentes farmacêuticas e saúde pública: desafios à política brasileira de acesso ao tratamento anti-retroviral. Cad. Saúde Pública, Rio de Janeiro, v. 24, n. 7, p. 1.4671.478, jul, 2008.

MORAES, M. F. V. et al. Epidemiological profile of tuberculosis cases in a priority municipality of the state of Maranhão. Rev. Pesq. Saúde, [S.l.], v. 18, n. 3, p. 147-150, set.-dez., 2017.

MUELLER, S. P. M.; PERUCCHI, V. Universidades e a produção de patentes: tópicos de interesse para o estudioso da informação tecnológica. Perspect. Ciênc. Inf, Belo Horizonte, v. 19 n .2, apr.jun. 2014.

NETO, M. S. et al. Clinical and epidemiological profile and prevalence of tuberculosis/HIV coinfection in a regional health district in the state of Maranhão, Brazil. J. Bras. Pneumol., [S.l.], v. 38, n. 6, p. 724-732, 2012.

NETTO, A. R. Tuberculosis: the neglected calamity. Revista da Sociedade Brasileira de Medicina Tropical, Uberaba, v. 35, n.1, p. 51-58, jan.-fev. 2002.

OMS - ORGANIZAÇÃO MUNDIAL DA SAÚDE. Plano de ação para a prevenção e controle da tuberculose. Washington, D. C., EUA: OMS, 2015. CD54/11, Rev. 1.

PEDRO, H. S. P. et al. Cenário atual da tuberculose. Hansen Int. [S.I.], v. 39, n. 1, p. 40-55, 2014.

PIERI, F. A.; MUSSI, M. C.; MOREIRA, M. A. S. Óleo de copaíba (Copaifera sp.): histórico, extração, aplicações industriais e propriedades medicinais. Rev. Bras. Plantas Med., [S.I.], v. 11, n. 4, p. 465472, 2009.

RABAHI, M. F. et al. Tratamento da tuberculose. J. Bras. Pneumol., [S.l.], v. 43, n. 5, p. 472-486, 2017.

REIS, J. G. et al. Epidemiology and health services: 25 years in review. Epidemiol. Serv. Saúde, Brasília, v. 26, n. 4, p. 685-700, 2017.

RIBEIRO, D. A. et al. Promising medicinal plants for bioprospection in a Cerrado area of Chapada do Araripe, Northeastern Brazil. Journal of Ethnopharmacology, [S.l.], v. 155, p. 1.522-1.533, 2014.

SANTANA, L. C. L. R.; MACHADO, K. C.; FREITAS, R. M. Prospecção científica e tecnológica da Mikania glomerata sprengel. Revista GEINTEC, [S.l.], v. 4, n. 3, p. 1.026-1.034, 2014.

SILVA, A. N. et al. Aumentar a atividade antituberculose em labdano Diterpenos de Copaifera oleoresin por meio de modificação estrutural. J. Braz. Chem. Soe, [S.I.], v. 28, n. 6, p. 1.1061.112, 2017.

SILVA, A. R.; SOUSA, A. I.; SANT'ANNA, C. C. Care practices employed in the treatment of children and adolescents with latent tuberculosis infection. Epidemiol. Serv. Saúde, Brasília, v. 23, n. 3, p. 547-552, jul.-set. 2014. 
SILVA, E. M. S. et al. Metabólitos de endofíticos Aspergillus fumigatus e sua em vitro efeito contra o agente causador da tuberculose. Acta amazônica, [S.l.], v. 48, n. 1, p. 63-69, 2018.

SILVA, P. F.; MOURA, G. S.; CALDAS, A. J. M. Fatores associados ao abandono do tratamento da tuberculose pulmonar no Maranhão, Brasil, no período de 2001 a 2010. Cad. Saúde Pública, Rio de Janeiro, v. 30, n. 8, p. 1.745-1.754, ago. 2014.

SOARES, J. G. et al. Estudo químico de óleos essenciais, oleaginosas e láticas da Amazônia I. Composição e oxidação do óleo de uma espécie de Copaifera. Acta Amazonica, [S.l.], v. 9, p. 6559, 2003.

SOUZA, D. Demora na concessão de patentes desestimula a inovação industrial. American Chamber of commerce for Brasil. [S.l.: s.n], 2015.

TINCUSI, B. M. et al. Antimicrobial terpenoids from the oleoresin of the Peruvian medicinal plant Copaifera paupera. Plant. Med., [S.l.], v. 68, n. 9, p. 808-812, 2002.

VEIGA JUNIOR, V. F.; PINTO, A. C. O Gênero Copaifera L. Química Nova, [S.I.], v. 25, n. 2, p. 273-286, 2002.

ZAGMIGNAN, A. et al. Caracterização epidemiológica da tuberculose pulmonar no Estado do Maranhão, entre o período de 2008 a 2014. Rev. Investig. Bioméd., [S.l.], v. 6, p. 6-13, 2014.

\section{Sobre os Autores}

\section{Vitória Santos Azevedo}

E-mail: azevedov614@gmail.com

Graduanda em Ciências Naturais/Química.

Endereço profissional: Av. Aurila Maria Santos Barros de Sousa, s/n, Bairro: Loteamento Frei Alberto Beretta, Grajaú, MA, CEP: 65940-000.

\section{Adrielle de Sá Araújo}

E-mail: adriellesa2016@gmail.com

Graduanda em Ciências Naturais/Química.

Endereço profissional: Av. Aurila Maria Santos Barros de Sousa, s/n, Bairro: Loteamento Frei Alberto Beretta, Grajaú, MA, CEP: 65940-000.

\section{Marcelo Borges de Sousa}

E-mail: marcelloborgges1@gmail.com

Graduando em Ciências Naturais/Química.

Endereço profissional: Av. Aurila Maria Santos Barros de Sousa, s/n, Bairro: Loteamento Frei Alberto Beretta, Grajaú, MA, CEP: 65940-000.

\section{Marcos dos Santos Barros}

E-mail: santtosmarcos68@gmail.com

Graduando em Ciências Naturais/Química.

Endereço profissional: Av. Aurila Maria Santos Barros de Sousa, s/n, Bairro: Loteamento Frei Alberto Beretta, Grajaú, MA, CEP: 65940-000. 


\section{Jefferson Almeida Rocha}

E-mail: jeffersonbiotec@gmail.com

Doutor em Biotecnologia.

Endereço profissional: Campus de São Bernardo, Rua Projetada, s/n, Bairro Planalto, São Planalto, MA, CEP: 65550-000. 